\title{
Percepción en la salud bucal de adolescentes embarazadas
}

\author{
Maria Luiza da Matta Felisberto Fernandes ${ }^{1}$ \\ Patrícia Corrêa-Faria ${ }^{2}$ \\ Bertha Angélica Chávez González ${ }^{3}$ \\ Laura Helena Pereira Machado Martins ${ }^{4}$ \\ Saul Martins Paiva ${ }^{5}$ \\ Isabela Almeida Pordeus ${ }^{6}$
}

\section{Resumen}

Objetivo: Verificar la interferencia del embarazo en las percepciones en salud bucal de adolescentes embarazadas de 12 a 18 años, con baja condición socio-económica, residentes en Belo Horizonte, Minas Gerais. Material y Métodos: Se realizó un estudio cualitativo con las adolescentes $(n=60)$ divididas en dos grupos: embarazada $(n=30)$ o no embarazada $(n=30)$. Entre las gestantes, diez estaban en el primer trimestre de gestación, trece en el segundo, y siete en el último trimestre de gestación. Los datos fueron recolectados a través de entrevistas individuales.
Se realizó el análisis del contenido. Resultados: Tres categorías de análisis surgieron a través del contenido manifestado en ambos grupos: consciencia de determinantes de la salud bucal (conocimientos y actitudes), reacciones emocionales relativas a la salud bucal (sentimientos positivos, pasividad y sentimientos negativos) y valores en salud bucal (apariencia, función social de los padres). Conclusión: Entre las jóvenes se observó que la experiencia del embarazo no interfirió en las percepciones en salud bucal.

Palabras Claves: salud bucal, embarazo, adolescente.

Artigo Original

\section{Percepção em saúde bucal de gestantes adolescentes}

\section{Resumo}

Objetivo: verificar a interferencia da gravidez nas percepções em saúde bucal de adolescentes grávidas, com idades entre 12 e 18 anos, com baixa inserção sócio-econômica, residentes em Belo Horizonte, Minas Gerais. Método: Realizou-se um estudo qualitativo com adolescentes $(\mathrm{n}=60)$ divididas em dois grupos de acordo com a experiência de gravidez $(n=30)$ ou não $(n=30)$.

\footnotetext{
${ }^{1}$ MSc, Doutoranda em Odontologia (Odontopediatria), Universidade Federal de Minas Gerais, Belo Horizonte, Minas Gerais, Brasil e-mail: marialuizadamatta@gmail.com

${ }^{2}$ MSc, Doutoranda em Odontologia (Odontopediatria), Universidade Federal de Minas Gerais, Belo Horizonte, Minas Gerais, Brasil e-mail: HYPERLINK “mailto:patricia.faria08@yahoo.com.br" patricia.faria08@yahoo.com.br

${ }^{3}$ MSc, Doutoranda em Odontologia (Odontopediatria), Universidade Federal de Minas Gerais, Belo Horizonte, Minas Gerais, Brasil e-mail: HYPERLINK "mailto:angelicachavez2008@hotmail.com" angelicachavez2008@hotmail.com

${ }^{4}$ PhD, Universidade Federal de Minas Gerais, Belo Horizonte, Minas Gerais, Brasil

5 PhD, Professor Titular, Universidade Federal de Minas Gerais, Belo Horizonte, Minas Gerais, Brasil HYPERLINK "mailto:smpaiva@uol.com.br" smpaiva@uol.com.br

6 PhD, Professora Titular, Universidade Federal de Minas Gerais, Belo Horizonte, Minas Gerais, Brasil HYPERLINK

"mailto:isabelapordeus@odonto.ufmg.br"isabelapordeus@odonto.ufmg.br
} 
Entre as gestantes, dez estavam no primeiro trimestre de gravidez, 13 no segundo trimestre e sete no último trimestre de gestação. Os dados foram coletados através de entrevistas individuais. Realizou-se a análise de conteúdo. Resultados: Três categorais de análise emergiram através do conteúdo manifesto em ambos os grupos: consciências de determinantes da saúde bucal (conhecimentos e atitudes), reações emo- cionais relativas à saúde bucal (sentimentos positivos, passividade e sentimentos negativos) e valores em saúde bucal (aparência, função social dos pais). Conclusão: Entre as jovens observouse que a experiência da gravidez não interferiu nas percepções em saúde bucal.

Palavras Chave: saúde bucal, gravidez, adolescente.

\section{Original article}

\section{Perception in oral health of pregnant adolescents}

\begin{abstract}
Objective: Check the interference of pregnancy on perceptions of oral health in poor pregnant aged 12-18 years old living in Belo Horizonte, Minas Gerais. Methods: A qualitative study was carried with teenagers $(n=60)$ divided into two groups according to the pregnancy experience $(n=30)$ or not $(n=30)$. Among pregnant woman, 10 were in the first trimester, 13 in the second and seven in the last trimester of pregnancy. Data were collected through individual interviews. Content analysis was performed. Results: Three categories of the manifest content analysis appeared in both groups: awareness of oral health determinants (knowledge and attitudes), emotional reactions related to oral health (positive feelings, negative feelings and passivity) and values in oral health (appearance, social function of parents). Conclusion: Among the children analyzed, pregnancy experience did not influence the perceptions of oral health.
\end{abstract}

Keywords: oral health, pregnancy, adolescent.

\section{Introducción}

En Brasil se observa la reducción de los índices de fecundidad (1), sin embargo aún es expresi- vo el número de adolescentes embarazadas. De acuerdo con el último informe del Instituto Brasilero de Geografía y Estadística- IBGE (2), una de cada cinco mujeres embarazadas tenía hasta 19 años (21,15\% de las madres de nacidos vivos). Durante la adolescencia, la gestación es una situación preocupante, principalmente considerándo el aspecto social de la madre y del niño (3).

En la gestación hay una variedad de alteraciones fisiológicas, anatómicas y hormonales. Pueden ocurrir alteraciones en los sistemas cardiovascular, respiratoras y gastrointestinal, además de mayor susceptibilidad a las infecciones orales $(4,5)$. Entre las alteraciones bucales, están la gingivitis, el granuloma piogénico, erosión del esmalte dentario, xerostomía y movilidad dentaria (4). Es conocido también que en el Brasil, las enfermedades bucales son altamente prevalentes, no obstante la prevalencia no es distribuida igualmente entre la población brasileña. Personas de minoría étnica o aquellas económicamente desfavorecidas son afectadas desproporcionalmente por la caries, gingivitis y la enfermedad periodontal (6).

No hay un consenso en la literatura de las alteraciones bucales asociadas a la gestación. En la perspectiva del ciclo de vida, la adolescencia es un es- 
tadio crucial de desarrollo de estilos de vida y cuidados en salud que perdurarán en la edad adulta y transmitirán estos valores a sus descendientes (7). Por lo tanto, el objetivo de este estudio fue verificar la percepción en salud bucal en un grupo de adolescentes embarazadas e no embarazadas.

Se esperó observar una baja percepción de alteraciones bucales en el embarazo y pobres índices de conocimiento sobre salud bucal, ya que la prevalencia de las enfermedades bucales es aún alta en el grupo de adolescentes brasileñas de baja condición socio-económica.

\section{Material y métodos}

Fue realizado un estudio de naturaleza cualitativa con adolescentes gestantes y adolescentes que nunca estuvieron embarazadas, con edades entre 12 e 18 años, residentes en una zona periférica de la ciudad de Belo Horizonte, Minas Gerais, Brasil.

Fueron realizadas entrevistas individuales por una dentista previamente entrenada y capacitada. La investigación fue aprobada por el Comité de Ética en Pesquisa de la Universidad Federal de Minas Gerais, bajo el dictamen número ETIC084/01. Previamente a la entrevista, las adolescentes y responsables recibieron informaciones concernientes a los objetivos del estudio y firmaron un término de consentimiento informado.

\section{Diseño del estudio}

La investigación fue realizada en el Puesto de salud pública de la Villa San José. Esta es una de las áreas con más alto grado de vulnerabilidad social de la ciudad de Belo Horizonte, Minas Gerais, Brasil. Entre las 51 adolescentes atendidas en la unidad básica de salud, 30 participaron en la investigación hasta que los temas emergentes de análisis de discurso se saturaron, iniciándose una repetición de los mismos, indicando así la definición del tamaño de la muestra.

Fueron seleccionadas también 30 adolescentes sin historia de gestación y residentes en la misma región. Los grupos fueron clasificados de acuerdo a la edad.

Las entrevistas fueron realizadas en una sala específica de la Unidad Básica de Salud sin interferencia de factores externos como ruido $u$ otros factores que pudiesen desviar la atención de la entrevistada o de la entrevistadora.

Para realización de la entrevista fue utilizado un guión semi-estructurado compuesto por seis preguntas abiertas que podrían revelar diferentes aspectos de la percepción de las adolescentes sobre la salud bucal. Estas fueron : “Cuál es su punto de vista sobre el significado de la salud bucal?", “¿Cuál es su punto de vista de cómo cuidar de su salud?”, “¿Cuál es su punto de vista de cómo cuidar su boca, podría hablarme un poco sobre el dentista, quién o qué influenció en su punto de vista sobre salud bucal?”, “¿Madre con dentadura mala, el hijo, va tener mala dentadura?". Las gestantes fueron cuestionadas también sobre interferencias del embarazo en la salud bucal. Todas las entrevistas, que tuvieron un promedio de 40 minutos, fueron grabadas y transcritas para el proceso de análisis del contenido.

El estudio principal constó de una muestra por conveniencia de treinta adolescentes embarazadas de 12 a 18 años con un promedio de 15,6 años. Diez adolescentes estaban en el primer trimestre de gestación, doce en el segundo trimestre y ocho en el último trimestre de la gestación. Las entrevistas fueron numeradas (de 1 a 60) de manera aleatoria. Para diferenciar los subgrupos estudiados (gestantes y no gestantes), se adoptaran las siguientes abreviaciones: A para las adolescentes no gestantes y G para las gestantes. 


\section{Análisis de los datos}

Para el análisis cualitativo del contenido, el modelo de Graneheim \& Lundman (8) fue usado, creándose el significado de las unidades, códigos y categorías. Una decisión fundamental antes de realizar tales análisis, es definir si ella se debe centrar en los contenidos manifestados (componentes obvios y visibles) o latentes (significados implícitos). El presente artículo se concentró en el contenido explícito. El tema, categorías y subcategorías, fue creado después de la discusión entre todos los investigadores y revisado por una socióloga y otro investigador externo, con formación en el área de odontología y experiencia en este tipo de metodología. La saturación de códigos, dentro de cada categoría fue encontrada durante los análisis de las entrevistas con la recurrencia de los mismos códigos. La validad del método fue asegurada por condiciones de credibilidad, en que el objetivo, la selección y la coleta de los datos pueden ser revisados y presentados.

A partir de las entrevistas, el tema principal fue caracterizado como percepciones en salud bucal. Después de analizarse el material de las entrevistas, el contenido manifestado puede ser descrito en la forma de tres categorías y siete subcategorías asociadas, como se observa en la Tabla 1.

\section{Resultados}

\section{Contenidos relatados}

\section{Consciencias de determinantes de la salud bucal}

La principal categoría puede ser subcategorizada en conocimientos y actitudes. Para las entrevistadas salud es un tema que ellas no tienen expresado anteriormente, pero lo consideraban importante para estar bien consigo misma y no interferir en la fuerza de trabajo. La salud bucal no fue relacionada a la salud general en ningún momento como demuestran los relatos:

Para cuidar de mi salud yo voy al médico regularmente..., es bueno tener una boca que este bien, hace que estés más feliz y no perturba la salud (18 años, no embarazada).

Salud es que tú puedas trabajar, hacer las cosa en casas, ¿ir al médico para hacerse un chequeo es importante no? (18 años, embarazada)

Para el cuestionamiento sobre el significado de la salud bucal las respuestas fueron una descripción del conocimiento que ellas tenían y una relación de conductas que eran necesarias para una buena salud bucal, y fue primeramente relacionada a no tener mal aliento:

... yo trato más mi cuerpo que mi boca. La boca es la última yo me olvido mucho de cepillarme los dientes ¿el apuro no? Por ejemplo no puedo de dejar de peinarme porque eso aparece, la boca en cambio te comes un caramelo y listo no vas a tener mal aliento. (17 años, embarazada).

\section{Conocimientos de salud bucal}

La caries fue asociada por las adolescentes al consumo frecuente de caramelos y gaseosas y fue citada como la manifestación bucal más común entre las alteraciones bucales. A pesar de eso no fue percibida como una enfermedad por ninguno de los grupos de adolescentes, en la medida en que es común en el medio en que viven:

Yo no pongo a la caries como una enfermedad. Es una cosita de nada que no te perjudica porque es bien chiquita y la gente no la ve, no interfiere en las obligaciones, no es enfermedad... no es un problema de salud también, porque se puede arreglar. (A.C., 16 años, embarazada).

El único autocuidado relacionado a la salud bucal que las adolescentes reconocieron fue el cepillado. La palabra "solamente" refleja la idea de que apenas esta práctica ya sea suficiente para mantener la salud bucal. 
...yo me levanto y me cepillo los dientes, después del almuerzo yo me cepillo solamente y se acabó. No cuido mi boca solamente la mantengo limpia. Lo que hago más es bañarme, ponerme ropa limpia, mi boca yo solamente la cepillo (V.A., 15 años, embarazada).

El flúor fue citado también como medio de prevención, no obstante apenas cuando es aplicado por el profesional. A pesar que la mayoría de las pastas dentales comercializadas en el Brasil poseen flúor, este vehículo no fue citado como medio de prevención de la caries dentaria.

Mi hermano.... saca una cita con el dentista para que haga un tratamiento con flúor, él sacó la cita al inicio de mi embarazo, él me explica las cosas, que es importante mantener la salud y no perderla para no tener que pagar caro, para cuidar, entonces yo creo muy importante eso por lo que él me dice, por eso yo vine aquí hoy. (A.E., 16 años, embarazada).

Se observó en los relatos de las entrevistadas que a pesar de saber que los caramelos y las gaseosas están relacionados con la caríes, la concepción de reducir el consumo no es viable en virtud del mayor costo de otros tipos de alimentos:

En el colegio yo compro muchas gomas de mascar, muchos caramelos no aguanto estar sin comer, como yo no como refrigerio aquí en el colegio porque no me alcanza el dinero. Así yo me quedo con hambre y en ese momento chupo un caramelo. Yo salgo de mi casa a las 6 de la mañana y llego a las 11 y 30, yo como caramelo todo el tiempo, solo así yo puedo estudiar (S.E., 16 años, no embarazada).

Sobre las creencias de salud bucal durante el embarazo, el grupo de gestantes fue cuestionado si la gestación tuvo alguna influencia en las condiciones de salud bucal. No obstante, sorprendentemente, todas las adolescentes no juzgaron que el embarazo tuviese interferido en la condición de la salud bucal, independiente del mes gestacional, como se puede observar en el relato:

Durante en el embarazo se malogra solo cuando se toma mucho antibiótico. Porque cuando la mujer necesita tomar remedio cuando está esperando bebe, ahí que los dientes se malogran, pero es por causa del remedio. (G.R., 14 años, embarazada).

Hay una cultura popular de la cual hacen parte los cuidados con los dientes. Se observa que en la cultura de este grupo, hay conductas establecidas con relación a los medios de prevenir molestias en la boca y lo que se debe hacer en caso de dolor.

Pasta de diente en el hueco del diente es buena porque esteriliza el diente, o también gotear kerosene en el diente, y es bueno también colocar hielo. (A.M., 16 años, embarazada).

Sobre las fuentes de informaciones en salud bucal a través de los relatos de las adolescentes, se pudo observar la riqueza de informaciones sobre salud bucal que provenían de diversos medios como el ambiente familiar, escolar, profesional y los medios de comunicación. Sin embargo la madre fue la persona más citada como gran influencia en la percepción sobre la atención odontológica y los hábitos en salud bucal de los hijos, como demuestran los relatos a continuación:

Todo lo que yo sé lo aprendí con mi mamá, cuando yo era pequeña ella me decía a cada rato para cepillarme...aprendí con ella, ella es quien se preocupa conmigo. Creo que tuve oportunidad de aprender esas cosas (relativas al mantenimiento de la salud bucal) porque mi mamá me enseñó (A.R., 15 años, embarazada).

El colegio ejerció influencias opuestas entre las adolescentes entrevistadas con relación a la contribución en salud bucal. Al mismo tiempo en que algunas elogiaron el papel del colegio en la divulgación de los medios de prevención en salud bucal, otras criticaron la forma como fueron "impuestos" tales programas.

En la escuela yo iba y me cepillaba ellos solo mandan no enseñan. Entonces yo aprendi así, vi a mis primos cepillándose y los imité, y yo hago igual, de la escuela yo no aprendí (A. R. 15 años embarazada). 
En el colegio ellos solo nos mandaban a ir a cepillarnos. Cuando iba a hacer el cepillado en el colegio, yo me salía de la fila, yo no creía que era interesante. Ni yo ni las niñas de aquella época, ellos no hablaban de la importancia del cepillado de los dientes. Todo el mundo creía que dolía que te hacia herida, que te iba a quedar adolorido... (E.R., 15 años, embarazada).

Ya otros relatos revelan al profesor como alguien con quien los alumnos tienen más intimidad, confianza y atención:

La profesora de geografía decía que la gente se tenía que cepillar muchos los dientes, dos, tres veces por día... Ella hablaba que tenía muchas niñas bonitas con la boca fea, entonces yo me quede pensando que era conmigo y me comencé a cepillar más. (D.J., 18 años, embarazada).

En este estudio, el papel de los cirujanos dentistas, dentro de la multiplicidad de los medios de educación en salud bucal, fue confirmar las informaciones aprendidas en los otros ambientes como en los medios de comunicación:

Yo escucho siempre alguna cosa de cuidar los dientes y boca en la televisión Mucha prensa habla, más todavía en el colegio, en mi casa también mi madre habla. Las cosas que los otros hablan es bueno, mas a mí me gusta siempre de preguntar al dentista si es verdad (K.R., 16 años, embarazada).

\section{Actitudes}

Las adolescentes informaron que tenían el hábito de cepillarse los dientes como forma de mantener la salud bucal, sin embargo lo olvidaban muchas veces:

Yo me olvido mucho de cepillarme los dientes ies el apuro no? (L.S., 17 años, no embarazada).

A pesar de que el relato predominante de los motivos de ir al dentista se concentra apenas en los dolores y emergencias, en ambos grupos entrevistados, los relatos demuestran también la dificultad de acceso a los servicios públicos odontológicos y la dificultad financiera que aleja la posibilidad de acceder a un atendimiento privado.

... Yo vine aquí para sacar una cita pero no consegui, me dijeron que solo se consigue de mañana. Pero yo me levanto a las 6 de la mañana para ir a trabajar. No tengo miedo de dentista porque voy desde jovencita, lo que pasa es que no tengo a nadie que venga mañana para sacar cita por mí. (L.S., 17 años, no embarazada).

La mayoría de las gestantes no buscan al dentista, pero las justificativas no incluyeron a la gestación como un impedimento para la búsqueda de la atención odontológica. Las adolescentes embarazadas entrevistadas no creen que la gestación fuese una barrera o impedimento a la atención odontológica:

Ganas yo hasta tengo de ir al dentista para que él cuide bien de mi boca... No tengo miedo del dentista, es pereza (R.S., 15 años, embarazada).

... mujer embarazada no puede ir al dentista, mi tía y mi madre me dijeron eso. Porque produce un daño al bebe. Ellas hablan un montón de cosas, pero yo voy, ellas no saben de nada. (S.B., 15 años, embarazada).

La falta de información y la prioridad secundaria con relación a la salud bucal fueron citadas como impedimento para que las adolescentes embarazadas busquen atención odontológica durante el embarazo:

Estaba pensando en ir al dentista pero después del embarazo. Porque ahora es mucha consulta, examen, tengo el colegio también no voy a tener tiempo. (G.C., 12 años, embarazada).

\section{Relaciones emocionales relacionadas a la sa- lud bucal}

Esta categoría describe los sentimientos de las informantes sobre aspectos relacionados a la sa- 
lud bucal, incluyendo tres subcategorías de sentimientos: positivos, impasibles e negativos.

\section{Sentimientos positivos}

A pesar que la gran mayoría de las entrevistadas de ambos grupos citaron actitudes de los profesionales como factores que las alejan de la atención odontológica, hubo quien relató sentimientos positivos en hacerse consultas odontológicas:

... yo creo bueno ir al dentista, porque siempre cuando voy ellos hablan que mis dientes están buenísimos. Entonces me siento elogiada. No tengo nada de miedo porque siempre que voy es para que ellos hablen bien de mí, no es para que lo curen. (F.N., 18 años, no embarazada).

El hecho de conocer las dificultades económicas para la recuperación da salud bucal, despertó en algunas, interés por la prevención y valorización de la salud bucal; el ambiente familiar fue determinante para esta actitud.

Mi hermano, como mi familia es pobre, siempre tuvo ganas de aprender. El saca una cita con el dentista, para que yo me ponga flúor, el me sacó una cita al inicio del embarazo, él me explica las cosas, que es importante mantener la salud, para no tener que pagar caro para cuidarla, entonces yo creo que es muy importante eso por causa de él, por eso yo vine aquí hoy (A.E., 16 años, embarazada).

\section{Sentimientos negativos}

El miedo al dentista fue relatado en ambos grupos. En este estudio se constató que uno de los orígenes de los miedos en Odontología, se relaciona a la falta de comprensión de los objetivos de la intervención, propiciado por un relato extremamente técnico del profesional.

Yo tengo pavor de llegar allá al dentista, ellos van hablando complicado, entonces tu piensan que tiene una cosa grave... (J.V., 17 años, no embarazada).
Otro origen de los miedos con relación al tratamiento odontológico, citado por las adolescentes de ambos grupos, fue el aspecto de los instrumentales odontológicos, además de la falta de información al paciente de la función de los instrumentales.

... El dentista es bueno para la salud pero es feo ir. No si creo que tengo miedo. Y sus maquinas... por Dios (S.A., 13 años, embarazada).

La mayoría de las entrevistadas abordaron la relación paciente-profesional como un factor de interferencia negativa en la búsqueda por la atención odontológica:

Yo no creo que sea muy bueno ir al dentista. Hay dentistas que no son educados. No saben conversar con las personas. Entonces yo no me animo a ir al dentista. (A.E., 16 años, embarazada).

Si es para que yo vaya la dentista hasta voy, pero el dentista podía tener una cara más gentil... (F.C., 16 años, no embarazada).

Se nota que el recelo en buscar atención odontológica estaba bastante presente en los dos grupos de adolescentes, y la única causa que no fue común a los dos grupos fue la correlación de la figura del dentista a la del obstetra conforme se manifestó en la conversación de una adolescente embarazada. Tal vez la aproximación del parto haya interferido en la angustia presentada por esta gestante pudiendo estar desencadenando el stress observado.

Tengo miedo del médico, de la boca y de lo que me saque, Ay Dios (L.S., 15 años, embarazada).

La pereza y la inseguridad fueron los sentimientos que más interfirieron en la busca del cuidado y / o recuperación de la salud bucal:

Ya pensé mucho en ir al dentista, pero no podía... yo no sabía cómo hablar, como conversar con las personas. Hoy 
mi hermana me llamó, y sacó el turno para que yo venga aqui. Nunca fui al dentista porque no sé, a mi no me gusta ir solita a ningún lugar. (M.E., 15 años, no embarazada).

Otro sentimiento negativo fue expresado a través de los relatos que indican la incapacidad de mantener la salud bucal a través exclusivamente de las acciones propias del individuo siendo necesaria la participación del dentista

El dentista es el único que puede cuidar bien de la salud bucal de la boca de las personas. Para tener dientes buenos. Yo solamente consigo cepillarme los dientes, pero solita no se puede tener los dientes buenos, yo no se porque pero tengo que ir al dentista (R.C., 17 años, embarazada).

La influencia de la opinión de terceros fue vista y sentida como factor dificultador en el compromiso en acciones de mantención y promoción de salud bucal:

... No sé, dicen que allá es feo, que ellos tiene una máquina para hacer doler que ellos colocan el diente, así me dijo una chica de vive cerca de mi casa (A.F., 14 años, no embarazada).

Tienen que sacar de la cabeza de la gente esa idea de que el dentista es pesado. Todo el mundo dice que el dentista es malo y da miedo. Mucha gente tiene miedo del dentista por culpa de su familia y no va no? (A.R., 15 anos, embarazada).

Se constató por fin, a través de relato de las adolescentes, que las expectativas de tratamiento no correspondidas ejercen un efecto negativo para la busca de la atención odontológica:

Esa idea Yo fui al dentista porque estaba doliendo mucho, fui a ver si me arrancaba el diente pero lo que me dio fue la dipirona, y no sirvió. La próxima vez yo nunca volveré. (S.E., 16 años, no embarazada).

\section{Valores en salud bucal}

Esta categoría fue formada a partir de dos subcategorías: apariencia y función social de los padres para conquistarse la salud bucal.
La estética representó salud y armonía siendo un motivo muy atrayente para buscar al dentista. Sin embargo la función fue subyugada revelando-se a importancia dada à presencia dos dientes anteriores. Los padres, principalmente la madre fue extremamente valorizada como la persona capaz de ayudar en el cuidado o recuperación de la salud bucal.

\section{Apariencia/función}

La autoimagen fue muy valorizada y un objeto muy observado y controlado por ambos grupos de estudio, sin embargo la pérdida de los dientes anteriores fue percibida de manera más dramática que los dientes posteriores. La boca, los dientes, la sonrisa adquirieron un lugar de auto referencia y criterio de aceptación social.

Tiene que ir al dentista para tener dientes, hace parte del ser humano, tiene que tener diente ipero es por la vanidad no? ¿Perder un diente de adelante te imaginas? Atrás puede caer todo Pero adelante no. (R.M., 14 años, embarazada).

La perdida de los dientes no fue relacionada a la enfermedad. Se observó, también, que delante de las dificultades financieras la extracción fue considerada una solución:

... sacarte el diente está bien. Porque así, la niña está con diente malogrado, queda con el diente malogrado al muchacho no le va a gustar ¿no es así? Va tener que sacarlo, así si se lo saca está bien Así queda más bonita la sonrisa de la persona. El diente de adelante cuando no está ¿una se queda más fea no? Porque atrás nadie va a ver, en la parte de adelante todo el mundo va a ver, mas estropeado es peor, es sucio (K.N., 18 años, no embarazada).

Se observó, también la creencia de que la perdida de los dientes es inevitable en algún momento de la vida:

“...No hay forma de que una persona tenga la boca buena siempre, sin problema ninguno no. Un día eso se acaba. 
Cuando tu estés viejo no vas a tener dientes, porque todos los viejos no tienen dientes, eso no es natural, no importa, siempre te llega la hora". (A.D., 17 años, embarazada).

\section{Función social de los padres}

Otro aspecto que quedo evidente en los relatos fue la responsabilidad de la mujer en los asuntos relativos a la salud en general, cuidados, educación y prevención.

El grupo apuntó a la manutención de la salud bucal como tarea de las "madres", existiendo un reclamo social cuando las propias madres fallaron en orientarlas sobre el asunto.

Dejaron clara la posición de que las madres ocupan un papel importante como principales agentes del cuidado en salud bucal de los hijos, y que aceptaban ese papel social. A los hombres no les fue aplicada esa responsabilidad al referirse a asuntos de salud o educación.

La madre tiene que cuidar de la boca del hijo, enseñarle a cepillarse, ayudarlo a cepillarse, llevarlo al dentista, porque la madre tiene paciencia y el padre es bruto y no tiene paciencia. (M.S., 13 años, no embarazada).

La emancipación de la mujer no desvinculó la responsabilidad femenina en el cuidado de la salud de los hijos. En este estudio se percibió también la exigencia y el reconocimiento de este papel social de la mujer.

... mi diente es débil, siempre da caries porque mi madre no me cuidaba bien. Ella trabajaba en la calle pero tenía que cuidar de mi también (E.R., 15 años, embarazada).

...Mi madre me llevó, y a mis hermanos, para que nos arreglen los dientes ella resuelve los problemas. Así es, la madre debe vigilar,..., llevar al dentista. Los dentistas ayudan mucho y al mismo tiempo ayuda poco, porque el niño está con la madre desde pequeña. El padre la mayoría trabaja, está afuera, no tiene tiempo de cuidar nada de los hijos. (A.F., 14 años, no embarazada).

\section{Discusión}

El presente estudio verificó la percepción en salud bucal de adolescentes embarazadas y no embarazadas, y la relación entre la gestación y alteraciones bucales. Este es un estudio cualitativo y fue realizado con una muestra de conveniencia obtenida de una unidad de salud. El estudio cualitativo nos brinda comprensión en profundidad de un tema estudiado y los resultados, en conjunto con otras fuentes de información, pueden ser útiles para una planificación efectiva de las intervenciones educativas.

Se consideró la percepción de las adolescentes sobre la salud bucal, se observó que a pesar de los relatos colectivos, citar la presencia de alteraciones bucales como la caries, falta de dientes o la alteración de hálito, estas no fueron descritas como enfermedad. Es válido reflejar que a pesar de que las adolescentes demostraron responsabilidad personal por la presencia de caries, parece haber una razón común para que esta se establezca demostrando también una impotencia delante de las alteraciones bucales citadas, que en la concepción de salud/enfermedad no es vista como enfermedad. Los relatos reproducen una culpa por el estado de la salud bucal y una impotencia en cambiar el cuadro establecido. Los modelos empleados para promover la salud bucal, culpan al enfermo por sus enfermedades debido a su comportamiento, encubriendo el fracaso de las políticas de salud en lidiar con factores sociales, económicos y ambientales inherentes al proceso salud/enfermedad (9).

La relación de mejores niveles de instrucción y una mejor percepción sobre las condiciones bucales está bien establecida (10). Luego, tratándose de una clase social menos favorecida, es de esperar que los factores sociales y culturales también interfiriesen en las percepciones de sa- 
lud y enfermedad. En Brasil, las perdidas dentarias están fuertemente asociadas a las cuestiones sociales y económicas (6), sin embargo cuando esas pérdidas ocurren en la región anterior de la cavidad bucal, el grupo estudiado no se conformó con la situación Lo mismo fue percibido en estudios de otras comunidades brasileñas, inclusive en comunidades rurales (11). La perdida de dientes se relaciona con componentes psíquicos y sociales importantes. En el Brasil, la apariencia es condición importante para la aceptación social, influenciando también en las oportunidades de trabajo. Así, la pérdida de dientes que pueda afectar la sonrisa, considerado un aspecto estéticamente agradable, convierte a las personas vulnerables a los sentimientos de inferioridad, rechazados e inseguros. Luego la pérdida dentaria impone a las personas de una situación social desfavorable $(12,13)$. En este estudio, así como verificaron Abreu et al (11), existió un confronto entre la realidad vivida (en la cual hay personas perdiendo dientes) y la realidad deseada (en la cual la perdida de dientes no esté presente). Ese confronto puede ser un aspecto importante para que los cambios se presenten. Esta misma realidad no es vivida en poblaciones asistidas económicamente $\mathrm{o}$ a través de amplias coberturas de planes de salud (14). Comparándose a las percepciones y hábitos en salud bucal de las adolescentes embarazadas y no embarazadas se observó que el grupo de gestantes no percibió alteraciones en sus condiciones bucales en la gestación. También no hubo diferencias de creencias, hábitos o prácticas en salud bucal. Sin embargo la literatura es muy clara en la descripción de las alteraciones que ocurren en la cavidad bucal durante la gestación. Mujeres embarazadas tiene una mayor incidencia de inflamación gingival comparadas a mujeres no embarazadas, la enfermedad periodontal también es más prevalente durante el embarazo, princi- palmente en grupos menos favorecidos económicamente $(15,16)$. Las razones para esta poca percepción de la interferencia del embarazo en la salud bucal pueden ser múltiples, incluyendo la inadecuada higiene bucal practicada por ambos grupos, el limitado acceso a los servicios odontológicos y los limitados conocimientos e instrucciones recibidas en salud bucal tanto a lo largo de la vida como en los cuidados prenatales $(17,18)$. Buerlein et al. (19) observaron que las prácticas y conocimientos adecuados en salud bucal de adolescentes embarazadas afro-americanas e hispano-americanas, de baja inserción social, estaban significativamente relacionadas con la mayor frecuencia de visitas al dentista cuando no estaban embarazadas.

Es importante resaltar la importancia del acceso al tratamiento odontológico y de las orientaciones en salud bucal para las gestantes: madres que reciben cuidados y orientaciones en salud bucal, tienden a cuidar mejor de la salud bucal de sus hijos, sea llevándolos a las consultas periódicas como instalando hábitos de higiene bucal y hábitos alimenticios favorables a la salud bucal; además de contribuir en la transmisión de una microflora bucal no patogénica a sus hijos $(16,19,20)$. Los conocimientos y comportamientos en salud bucal son importantes durante el embarazo, especialmente en grupos de riesgo como es considerada la adolescencia, para prevenir las enfermedades bucales o complicaciones sistémicas. En esta investigación, se atribuye a las madres el deber de ser la proveedora de la salud en la familia. Las madres fueron fuertemente exigidas en relación a esa obligación social, siendo culpadas por las condiciones de salud bucal de sus hijos. Este resultado está de acuerdo con otros trabajos $(3,11,12)$ y despierta la reflexión de la importancia de trabajarse con las gestantes y las otras adolescentes, madres en 
potencial para que transmitan creencias y valores favorables a la salud bucal.

No hubo diferencias marcadas en las percepciones en salud bucal entre las adolescentes gestante y no gestantes. Sin embargo se sabe que cuando un hijo es generado, hay una modificación en la identidad de los padres, los cuales transmiten para sus hijos madurez en que se encuentran. Un imaginario repleto de esperanza es construido por los padres (21). Creemos que en el escenario de extrema pobreza e incertidumbre de vivencias por las adolescentes embarazadas puede ser una explicación del porque no encontramos diferencias más marcadas en las manifestaciones sobre el tema salud bucal en el grupo das adolescentes embarazadas.

El estudio cualitativo no puede ser atribuido a todas las poblaciones ni generalizado a toda la sociedad brasileña. Sin embargo contribuye a la identificación de valores y prácticas en salud bucal. Tales conocimientos no tienen implicación apenas para intervenciones en salud bucal en el área estudiada, pero tiene implicaciones más amplias en la construcción de programas de intervención precoz para niños de baja edad y clase social. Esta es una necesidad en diversos países del mundo (3). Las únicas diferencias encontradas entre los grupos de adolescentes fue el miedo de médicos, así como el dentista, citado por una gestante. De esta forma analizamos que en este grupo, el embarazo no representó ningún impacto (positivo o negativo) en las percepciones en salud bucal. Investigaciones adicionales basadas en las investigaciones de conocimientos y prácticas en salud bucal pueden contribuir en la construcción de programas más efectivos en odontología social y preventiva. Por lo tanto, se sugiere el desarrollo de estudios con muestras representativas de la población, incluyendo adolescentes de diferentes clases sociales.

\section{Referencias}

1. Brasil. Ministério da saúde. Número de partos realizados em adolescentes de 10 a 19 anos no Brasil. Accesado (2011 Oct 10) Disponible en: http:/ / portal.saude.gov.br/portal/saude

2. IBGE. Jovens mães. Acessado (2012 Ago. 10). Disponible en: www.ibge.gov.br/ibgeteen/datas/saude/jovensmaes. html

3. Mahyar M, Leslie PZ, Gary RR. Oral health of early head start children: a qualitative study of staff, parents and pregnant women. Am J Public Health 2009; 99:245-251.

4. Giglio JA, Lanni SM; Laskin DM, et al. Oral Health Care for the Pregnant Patient Canadian Dental Association. JCDA. 2009;75:43-48

5. Giglio JA, Lanni SM; Laskin DM, et al. Oral health care for the pregnant patient. J Mich Dent Assoc. 2011;93:38-43.

6. Roncalli AG. National oral health survey in 2010 shows a major decrease in dental caries in Brazil. Cad Saude Publica. $2011 ; 27: 4-5$.

7. Jiang H, Petersen PE, Peng B, Tai B, Bian Z. Self-assessed dental health, oral health practices, and general health behaviors in Chinese urban adolescents. Acta Odontol Scand. 2005;63:343-352.

8. Graneheim UH, Lundman B. Qualitative content analysis in nursing research: concepts, procedures and measures to achieve trustworthiness. Nurse Educ Today 2004; 24:105-112.

9. Flores EMT, Drehmer TM. Knowledge, perceptions, behaviors and representations of oral health of teenagers of public schools of two neighborhoods of Porto Alegre. Cienc e saúde coletiva 2003; 8:743-752. 
10. Beneyto MY, Vera-Delgado MV, Pérez L, et al. Self-reported oral health and hygiene habits, dental decay, and periodontal condition among pregnant European women. Int J Gynaecol Obstet. 2011; 114:18-22.

11. Abreu MHNG, Pordeus IA, Modena CM. Representações sociais de saúde bucal entre mães no meio rural de Itaúna (MG), 2002. Ciênc. saúde coletiva 2005; 10: 245-259.

12. Weyant RJ, Manz M, Corby P, et al. Factors associated with parents' and adolescents' perceptions of oral health and need for dental treatment. Community Dent Oral Epidemiol 2007; 35:321-330.

13. Saliba-Garbin CA, Isper-Garbin AJ, Moreira-Arcieri R, et al., Adolescents' perception of oral health. Rev Salud Publica (Bogota). 2009; 11:268-277.

14. Shahrbanoo-Fadavi, Maureen C. Sevandal., Anne Koerber., et al. Survey of Oral Health Knowledge and Behavior of Pregnant Minority Adolescents. Pediatr Dent 2009 ; 31:405-408.

15. Lieff S, Boggess KA, Murtha AP, et al. The oral conditions and pregnancy study: periodontal status of a cohort of pregnant women. J Periodontol 2004; 75:116-126.

16. Boggess KA, MD, Diana M. Urlaub MPH, Massey KE, et al. Oral Hygiene Practices and Dental Service Utilization Among Pregnant Women JADA 2010; 141:553-56.

17. Wilder R, Robinson C, Jared H, Lieff S, Boggess K. Obstetricians' knowledge and practice behaviors concerning periodontal health and preterm delivery and low birth weight. J Dent Hyg 2007; 81:81.

18. Zanata RL, Fernandes KB, Navarro PS. Prenatal dental care: evaluation of professional knowledge of obstetricians and dentists in the cities of Londrina/PR and Bauru/SP, Brazil, 2004. J.Appl Oral Sci 2008; 16:194-200.

19. Buerlein JK., Horowitz AM., Child, WL. Perspectives of Maryland women regarding oral health during pregnancy and early childhood. J Public Health 2011; 71:131-135.

20. Greenberg BJ, Kumar JV, Stevenson H. Dental case management: increasing access to oral health care for families and children with low incomes. JADA 2008; 139:1114-1121.

21. Al Habashneh R, Guthmiller JM, Levy S, et al., Factors related to utilization of dental services during pregnancy. J Clin Periodontol 2005;32:815-821.

Correspondencia: Rua Rafael Magalhães 224, Santo Antônio. Belo Horizonte, Minas Gerais, Brasil; E-mail: marialuizadamatta@gmail.com Telefone: (31) 33422793

Recibido: 11 oct 2012

Aceptado: 02 nov 2012

Correspondencia: marialuizadamatta@gmail.com 